\title{
Lamp spectrum and spatial brightness at photopic levels: Investigating prediction using S/P ratio and gamut area
}

\author{
S Fotios $\mathrm{PhD}^{\mathrm{a}}$, D Atli MFA ${ }^{\mathrm{a}}$, C Cheal $\mathrm{PhD}^{\mathrm{a}}$ and $\mathrm{N}$ Hara $\mathrm{PhD}^{\mathrm{b}}$ \\ ${ }^{a}$ School of Architecture, University of Sheffield, Sheffield, UK \\ ${ }^{\mathrm{b}}$ Department of Architecture, Kansai University, Suita, Osaka, Japan
}

Received 17 February 2014; Revised 9 June 2014; Accepted 13 June 2014

An experiment was carried out to investigate spatial brightness at photopic levels under lighting of different spectral power distributions. One aim was to replicate the experiment reported in 1990 by Berman et al. demonstrating that light with a higher scotopic / photopic (S/P) ratio would be perceived as brighter. In addition, a third SPD was included to investigate gamut area and two additional procedures were employed to provide concurrent validity of the findings. It was concluded that while lighting of higher $S / P$ ratio was brighter, the $S / P$ ratio alone was insufficient to predict spatial brightness. A metric for the chromatic contribution is also needed, this being provided by gamut area in the current work.

\section{Introduction}

This paper discusses lamp spectral power distribution (SPD), spatial brightness and lighting for interior spaces at photopic levels of adaptation. Currently, a key strategic question for lighting is 'How can the consumption of electricity by lighting be reduced?'. A strong contender for the demand for rapid and major reductions in the electricity consumed by lighting is a reduction in the illuminances used ${ }^{2}$ and one approach to reducing illuminance is to use SPDs that better match human vision.

The purpose of lighting is to give information: To enable the people in a space to perceive the nature of the space they are in, what other people are doing and what they have to accomplish in a task. ${ }^{3}$ In offices, as

Address for correspondence: Steve Fotios, School of Architecture, University of Sheffield, The Arts Tower, Western Bank, Sheffield, S10 2TN, UK.

E-mail: steve.fotios@sheffield.ac.uk with other work places, lighting is needed so that the occupants can see to carry out their work tasks quickly, accurately and easily. ${ }^{4}$ For visual performance, a reduction in illuminance will not have significant effect if task conditions lie within the plateau region of the performance versus illuminance relationship. For tasks of high contrast (greater than approximately 0.2 ) the relative visual performance (RVP) model suggests little difference in visual performance between background luminances of $50 \mathrm{~cd} / \mathrm{m}^{2}$ and $169 \mathrm{~cd} / \mathrm{m}^{2} .5$ These luminances equate to illuminances of approximately 200 lux to 650 lux (assuming a white paper background of reflectance 0.8 ), which covers the illuminances typical of interiors. ${ }^{6}$ In fact, RVP decreases only by a small amount if the luminance is reduced to $12 \mathrm{~cd} / \mathrm{m}^{2}$ (47 lux). Many office tasks are now carried out using display screens which, being self-luminous, would benefit from a reduction in general illumination. ${ }^{7}$

Spatial brightness is the visual sensation of the magnitude of the ambient lighting within 
an environment such as a room. ${ }^{8,9}$ If the illuminance were to be reduced too much, producing a dim, gloomy environment, this could lead to negative effects on mood and motivation. A field study by Akashi and Boyce ${ }^{10}$ demonstrated that it is possible to use SPD to maintain spatial brightness at lower illuminance. Akashi and Boyce recorded occupants' judgements of lighting in four offices over a period of several months. ${ }^{10}$ Initially, each office had similar lighting, a correlated colour temperature (CCT) of $3500 \mathrm{~K}$ and mean desk illuminances of 544 lux to 586 lux. After nine months, the illuminance in two offices were reduced by one third (by removal of one of the three lamps in each luminaire) and CCT was increased to $6500 \mathrm{~K}$ in two offices by replacement of the lamps. These changes were balanced so that one office was unchanged, one office had a reduction in illuminance and an increase in CCT, one office had only an increase in CCT and one office a reduction in illuminance only. Responses were recorded using questionnaires seeking a yes/no response to statements regarding the visual environment. In the office where illuminance was reduced, this led to a significant increase in judgements that the office appeared gloomy; in the office where the reduction in illuminance was accompanied by an increase in CCT, there was a significant reduction in judgements that the lighting was too dim. This study suggests a $33 \%$ reduction in illuminance was countered by increasing the CCT from $3500 \mathrm{~K}$ to $6500 \mathrm{~K}$. However, data from other studies suggest CCT is not a reliable metric for the effect of SPD on spatial brightness. ${ }^{11-13}$

One approach to establishing a metric for spatial brightness would be to gather evidence from past experimental studies and use these to determine a best fit model, as was done by Ware and Cowan ${ }^{14}$ for the brightness of small fields. Approximately 70 past studies have investigated relative spatial brightness under lighting of different SPD, from which a recent review ${ }^{9}$ suggests that 19 provide credible evidence. These tend to support the proposal that carefully chosen SPDs can be used to maintain spatial brightness at a lower illuminance. However, attempts to use those data to screen potential metrics for SPD and spatial brightness were hindered because each model led to similar conclusions regarding the relative brightnesses of different lamps, thus preventing discrimination between the models. ${ }^{15}$ Furthermore, it is difficult to establish the SPDs of lamps used in past studies, these being rarely reported. Therefore, this paper reports an experiment carried out to investigate two proposed metrics for characterising the effect of SPD on spatial brightness.

One proposed metric is the $\mathrm{S} / \mathrm{P}$ ratio, the ratio of the luminous output of a light source evaluated according to the CIE scotopic (S) spectral luminous efficiency function, $V^{\prime}(\lambda)$, to the luminous output evaluated according to the CIE photopic (P) spectral luminous efficiency function, $V(\lambda){ }^{16}$ This was first proposed by Berman et al. ${ }^{17}$ who purposefully compared two light sources of near-identical chromaticity (and hence equal cone photoreceptor excitation) but different $\mathrm{S} / \mathrm{P}$ ratio. It was concluded that lighting of higher $\mathrm{S} / \mathrm{P}$ ratio appears brighter. Following new findings in vision this was amended to a contribution from the intrinsically photosensitive retinal ganglion cells (ipRGC) ${ }^{18}$ and there is some independent evidence for this. ${ }^{19}$ The S/P ratio also appears to correlate with visual performance at photopic level ${ }^{20}$ with lighting of higher $\mathrm{S} / \mathrm{P}$ ratio being proposed as a means of maintaining visual efficiency at reduced illuminance. There are two limitations of the Berman et al. study; first, their method has not yet been repeated; second, the relative importance of chromatic and rod (or possibly ipRGC) contributions to spatial brightness is unknown, in particular when the environment departs from the achromatic environment used by Berman et al. 
Spatial brightness will vary under lighting of similar illuminance but using different SPD because, while illuminance is defined by $\mathrm{V}(\lambda)$, spatial brightness exhibits a different spectral sensitivity. At the post-receptoral stage the visual system is organised in three channels. One is the achromatic channel where signals from the long- and medium-wavelength sensitive cone types are combined, and two are colour channels where the differences between signals from different combinations of cone types are taken. ${ }^{21} \mathrm{~V}(\lambda)$ was based on data collected primarily using flicker photometry and step-by-step brightness matching, techniques that tend to minimize activity in the colour channels, and $\mathrm{V}(\lambda)$ is hence considered to model the sensitivity of the achromatic channel only; brightness perception is dependent on activity in all three channels. ${ }^{22-24}$

One approach to predicting the chromatic contribution to brightness is to use standard metrics of lamp colour characteristics, for example CCT or CIE General Colour Rendering Index $\left(\mathrm{R}_{\mathrm{a}}\right)$. Past evaluation of such metrics suggests that gamut area is the most promising, ${ }^{11}$ as it correlated well with judgements of visual appearance of a lit scene using a matching task, and visual appearance may be considered a proxy for spatial brightness judgements. ${ }^{25}$ Gamut area is an area of colour space derived from the u',v' chromaticity coordinates of the eight colour samples used in the CIE General Colour Rendering Index when these are illuminated by the particular light source. It provides a measure of the colour differences between a range of coloured surfaces, with a larger gamut area implying greater saturation of surface colours, and thus possibly that the lighting is brighter. $^{11}$

This paper presents an experiment carried out to investigate spatial brightness under lighting of different SPD. The objectives were

- To validate the findings of Berman et al.., ${ }^{17}$ first by repeating their procedure and second by using an alternative procedure as used by Fotios and Cheal. ${ }^{26}$

- To determine whether the findings of Berman et al. vary if colour is introduced into the achromatic test environment.

- To add a third SPD, thus to investigate spatial brightness using sources of equal S/ $\mathrm{P}$ ratio but different chromaticity and gamut area.

This focus on the $\mathrm{S} / \mathrm{P}$ ratio and gamut area is not intended to imply that these are the best models for spatial brightness nor that they are all that is needed. Rather, this is intended to explore proposals raised in previous work.

\section{Method}

\subsection{Light source}

Three SPDs were generated for these trials using an LED array, designed and constructed by John Barbur and colleagues at City University, London. This comprised two identical, linear arrays of LEDs, with each array containing six clusters of four types of LED having different chromaticities (Table 1). The control system allowed the intensity of each type of LED to be independently modulated, thus allowing a wide range of unique spectra to be set. Of particular note for the current work, the four-LED system allowed for the $\mathrm{S} / \mathrm{P}$ ratio to be varied whilst maintaining a constant chromaticity. The LED arrays were fitted to the test booth above the position of the observer's head, and thus there was no direct sight of the light source.

The current work required three different SPDs, two having identical chromaticity but different $\mathrm{S} / \mathrm{P}$ ratios, and a third having similar $\mathrm{S} / \mathrm{P}$ ratio but different chromaticity to one of the others. These three SPDs are identified in Figure 1 and Table 2 displays their chromaticities, $\mathrm{S} / \mathrm{P}$ ratios and gamut areas. The values in Table 2 were derived from the SPDs measured from the observers' view point, 
and are thus the lamp SPDs as modified by internal reflection in the test apparatus. Measurements were recorded using a Konica-Minolta CS1000 spectroradiometer, calibrated immediately prior to this experiment.

Following Berman et al., two spectra were used having identical chromaticity (ten degree $\mathrm{x}, \mathrm{y}$ ) but different $\mathrm{S} / \mathrm{P}$ ratios ( $\mathrm{A}$ and $\mathrm{B}$ in Table 2). The third setting (C) was established

Table 1 Chromaticity coordinates of the LEDs used in the array as determined in the CIE $x, y$ chromaticity diagram for $2^{\circ}$ fields

\begin{tabular}{lll}
\hline Primary LED & $\mathrm{x}$ & $\mathrm{y}$ \\
\hline Red & 0.698 & 0.302 \\
Green & 0.154 & 0.666 \\
Blue & 0.146 & 0.036 \\
Amber & 0.592 & 0.407 \\
\hline
\end{tabular}
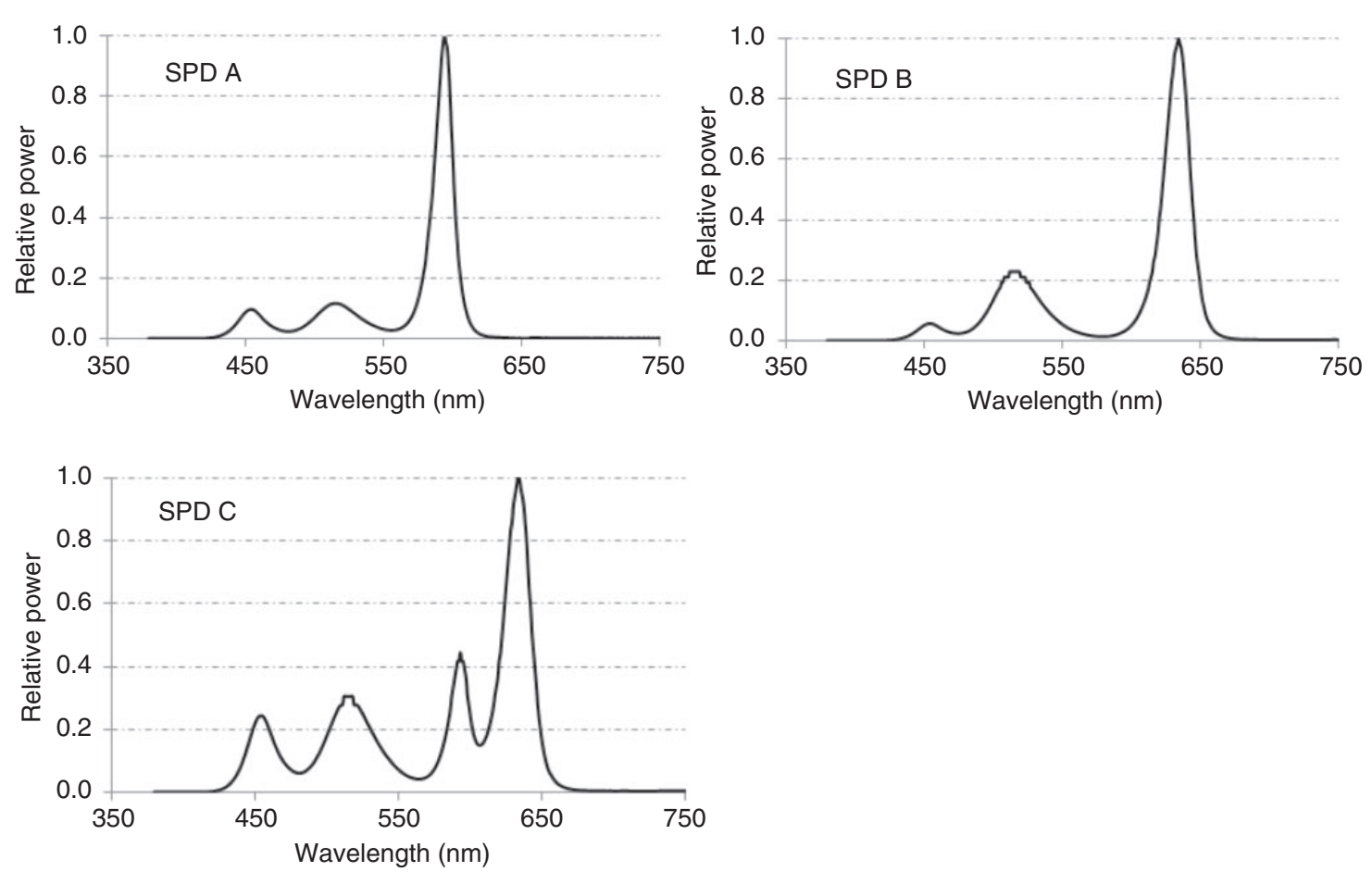

Figure 1 Spectral power distributions used. These were measured from the observers' view point and hence include modification by the test apparatus, and are normalised for a peak response of 1.0 by examining further spectra in the region of the chromaticity of A and B searching for the SPD having the largest gamut area and an $\mathrm{S} / \mathrm{P}$ ratio similar to that of $\mathrm{B}$.

Luminance was adjusted using two mechanisms. For the experimenter, this was done by using the control software to set a previously determined luminance. For test participants during the matching task, adjustment was carried out using a rotary dial, this having three $360^{\circ}$ turns from minimum to maximum to reduce the chance of a positional cue.

\subsection{Apparatus}

This experiment was carried out using the single booth shown in Figure 2, a similar apparatus to that used recently by Royer and Houser. ${ }^{27}$ The viewing chamber of the booth was of approximate dimensions $900 \mathrm{~mm}$ deep, 
$1000 \mathrm{~mm}$ wide and $1150 \mathrm{~mm}$ high. Test participants sat at the front of this booth at a distance approximately $700 \mathrm{~mm}$ from the rear wall and thus the sides extended behind their head, giving full field stimulation of the retina.

The interior surfaces were painted with a matt white paint having a reflectance of

Table 2 Description of the LED spectra and blended fluorescent lamps used in brightness assessments. For the current work, all properties were derived from SPDs measured from the observer's view of the test apparatus. Note: Berman et al. did not report S/P ratios: These were determined from the photopic and scotopic luminances reported in their Table 2

\begin{tabular}{lcccc}
\hline Light setting & $\times\left(10^{\circ}\right)$ & $\mathrm{y}\left(10^{\circ}\right)$ & $\mathrm{S} / \mathrm{P}$ & $\begin{array}{l}\text { Gamut } \\
\text { Area }\end{array}$ \\
\hline \multicolumn{2}{l}{ SPD used in current work } & & & \\
$\mathrm{A}$ & 0.49 & 0.40 & 1.02 & 0.0017 \\
$\mathrm{~B}$ & 0.49 & 0.40 & 1.77 & 0.0041 \\
$\mathrm{C}$ & 0.44 & 0.36 & 1.81 & 0.0069 \\
Lamps used by Berman et al. & & \\
R213 & 0.46 & 0.42 & 2.40 & - \\
WWG & 0.48 & 0.41 & 0.85 & - \\
\hline
\end{tabular}

approximately 0.8 , this being uniform across the visible spectrum. This environment was purposefully neutral, following Berman et al. Colour was introduced for some trials using a Mondrian array (Figure 2) covering the back wall of the booth. This array contained three colours (red, yellow and blue) of approximately equal proportions. Past results suggest that the degree of colourfulness does not significantly affect the results of spatial brightness judgements using category rating or matching procedures ${ }^{28}$ and thus there was no a-priori reason for the selection of these particular colours. When illuminated by a Verivide D65 daylight simulating light source these colour patches had the following CIE $2^{\circ}$ chromaticity coordinates: yellow, $\mathrm{x}=0.45$, $\mathrm{y}=0.44 ;$ blue, $\mathrm{x}=0.25, \mathrm{y}=0.26 ; \quad$ red, $\mathrm{x}=0.49, \mathrm{y}=0.32$.

It was important for the distribution of surface luminances to be stable under changes of SPD and luminance, i.e. that luminances measured at various points around the
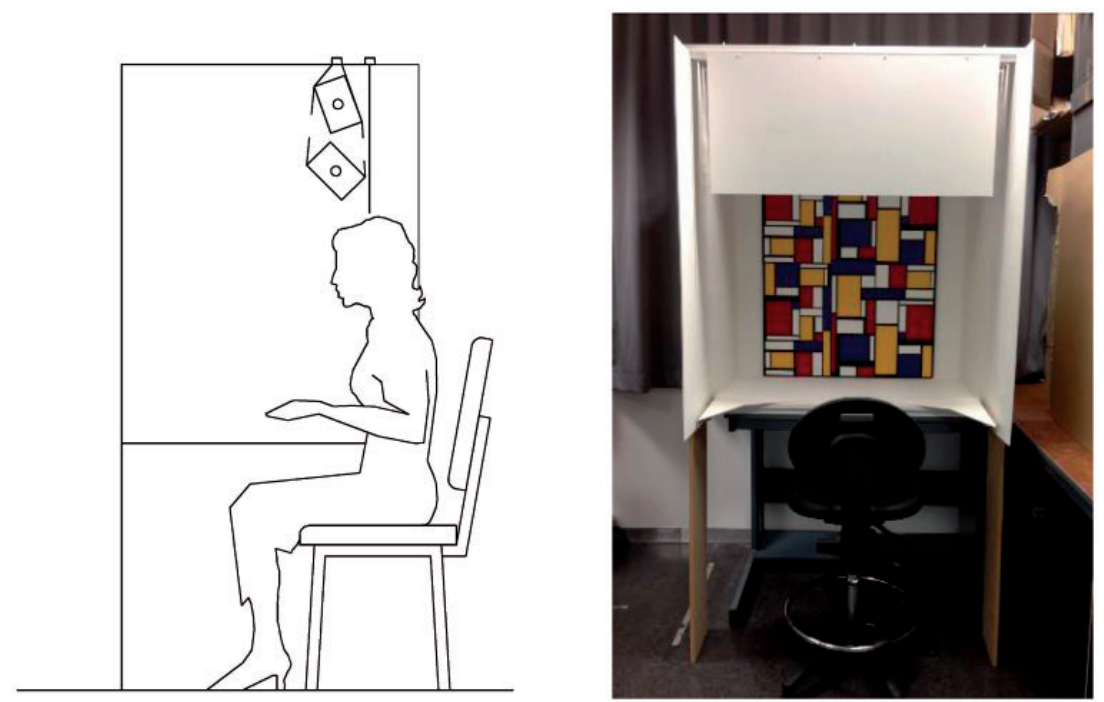

Figure 2 Section (not to scale) and photograph of the test apparatus. Note: for clarity in this photograph the test lighting is switched off and the laboratory lighting is switched on. This photograph also shows the Mondrian pattern on the back wall, introduced for the chromatic trials and removed for the achromatic trials 
cabinet interiors varied proportionally. Differences were not expected since all SPDs examined were provided by the same LED array. To assess the stability of the relative luminance distribution between different SPDs and luminance settings, luminances were measured at a grid of 26 points across the rear and side walls and floor of the booth, with a luminance meter (Konica-Minolta LS100 calibrated immediately prior to this experiment) aimed from the participant's viewpoint. With all SPDs and luminance settings, luminances at the sides of the rear wall were approximately $10 \%$ lower than that at the centre, and varied $20 \%$ from ceiling to floor.

\subsection{Procedure I: Berman et al.}

One stage of the experiment followed the procedure used by Berman et al. to compare the brightness of two light sources of identical chromaticity using full-field stimulation. The task followed, as near as possible, the procedure used by Berman et al. ${ }^{17}$ The key differences were

- In Berman et al. test participants were located inside a small room ( $2 \mathrm{~m}$ deep, $2 \mathrm{~m}$ wide and $2.3 \mathrm{~m}$ high). The LED array available did not offer sufficient power to light this environment to the same luminance and uniformity as in Berman et al. and hence a smaller space was used, as shown in Figure 2. This allowed full field stimulation, the critical requirement of Berman et al.
- An LED array was used rather than blended fluorescent lamps.

- The LED array did not enable the identical chromaticity to that used by Berman et al., but it is close (see Table 2). What was done was to ensure that the two SPDs used (A and B) were of the same chromaticity.

- The LED array did not permit as great a difference in $\mathrm{S} / \mathrm{P}$ ratio between the two blended fluorescent sources used by Berman et al. This was accounted for by using Brightness Lumens (see below) to predict the luminances required for equal brightness and resulted in a smaller luminance difference being used in trials than were used by Berman et al.

- An additional null condition with comparison between settings of the same SPD and luminance was added in order to better validate the procedure. In particular, whether the three successive presentations of each SPD was sufficient to offset interval bias. $^{29}$

Berman et al. used a sequential discrimination procedure to compare two SPDs. These were not compared on an equal luminance basis but with the luminances presented in four specific conditions (Table 3 ). In comparison 1, SPD B (high $\mathrm{S} / \mathrm{P}$ ratio) was presented at a lower luminance $\left(40 \mathrm{~cd} / \mathrm{m}^{2}\right)$ than was SPD A (low S/P ratio: $47 \mathrm{~cd} / \mathrm{m}^{2}$ ) to demonstrate that test participants would tend to report SPD B as brighter than A despite the lower luminance. Comparison 2 repeated comparison 1, using the same ratio of photopic luminances, but at a higher absolute

Table 3 Lighting conditions examined in the stages of experiment repeating Berman et $a l^{17}$

\begin{tabular}{|c|c|c|c|c|c|}
\hline & Comparison 1 & Comparison 2 & Comparison 3 & Comparison 4 & Comparison 5 \\
\hline & $A / B$ & $A / B$ & $A / B$ & $B / B$ & $A / A$ \\
\hline $\begin{array}{l}\text { Photopic luminances }\left(\mathrm{cd} / \mathrm{m}^{2}\right) \\
\text { Luminance ratio (higher /lower) } \\
\text { Predicted brighter setting }\end{array}$ & $\begin{array}{l}47 / 40 \\
1.18 \\
B\end{array}$ & $\begin{array}{l}67 / 57 \\
1.18 \\
B\end{array}$ & $\begin{array}{l}67 / 40 \\
1.68 \\
\text { A }\end{array}$ & $\begin{array}{l}67 \text { / } 57 \\
1.18 \\
\text { Higher } \\
\text { luminance }\end{array}$ & $\begin{array}{l}47 / 47 \\
1.00 \\
\text { Equal }\end{array}$ \\
\hline
\end{tabular}


luminance (67 and $57 \mathrm{~cd} / \mathrm{m}^{2}$ for $A$ and $B$ respectively), thus to examine spatial brightness at two typical interior light levels. Note that luminances reported here were as measured on the rear wall of the booth, at the centre point $700 \mathrm{~mm}$ above the floor, approximately the observer's view point if looking straight ahead. With the Mondrian in place, this point fell on the black border between adjacent white and blue patches. Test luminances were measured when the booth surfaces were achromatic and these same light settings were then also used when the Mondrian was inserted, thus maintaining the same horizontal illuminance following the approach used in previous work. ${ }^{12}$

The luminances at which SPDs A and B would appear equally bright were predicted using Brightness Lumens (Equation 1), a tentative metric for the effect of lamp spectrum on spatial brightness. ${ }^{30}$ Spaces lit by two different lamps of equal brightness lumens should appear equally bright

$$
\text { Brightness Lumens }=\mathrm{P}(\mathrm{S} / \mathrm{P})^{0.5}
$$

where, $\mathrm{P}$ and $\mathrm{S}$ are the luminous output of a light source evaluated according to the scotopic (S) spectral luminous efficiency function, $\mathrm{V}^{\prime}(\lambda)$ and the photopic $(\mathrm{P})$ spectral luminous efficiency function, $\mathrm{V}(\lambda)$.

We chose SPD A with a photopic luminance of $67 \mathrm{~cd} / \mathrm{m}^{2}$ as the reference, this being the luminance as used by Berman et al. for their low $\mathrm{S} / \mathrm{P}$ source in comparison 2 . According to brightness lumens, SPD B requires a photopic luminance of $51 \mathrm{~cd} / \mathrm{m}^{2}$ for equal brightness (a photopic luminance ratio of $67 / 51=1.31$ ). To promote a tendency for SPD B to be identified as brighter, this was presented in comparison 2 at a luminance of $57 \mathrm{~cd} / \mathrm{m}^{2}$, slightly above that needed for equal brightness but still a lower photopic luminance than SPD A.

In comparison $3 \mathrm{SPD} B$ (high $\mathrm{S} / \mathrm{P}$ ratio) was presented at a much lower luminance $\left(40 \mathrm{~cd} / \mathrm{m}^{2}\right)$ than was SPD A (low $\mathrm{S} / \mathrm{P}$ ratio: $67 \mathrm{~cd} / \mathrm{m}^{2}$ ), a luminance ratio of 1.68 compared with the ratio of 1.18 used in comparisons 1 and 2. In this situation, it was expected that test participants would tend to identify SPD A as brighter, the higher luminance of SPD A now outweighing the higher $\mathrm{S} / \mathrm{P}$ ratio of SPD B. In comparison 4 the two stimuli compared were of identical spectra (SPD B) but different luminances, the ratio (1.18) being similar to that as used in comparisons 1 and 2 . This is in effect a control comparison which examines whether the luminance differences used in comparisons 1 and 2 are discriminable. For this study an additional comparison was added, comparison 5, a null condition in which both settings had identical SPD and luminance.

In a trial, each light source was presented for 5 seconds, with three presentations of each source separated by a $100 \mathrm{~ms}$ dark interval. For each of the five comparisons, the two stimuli were evaluated ten times. Presentation order was counterbalanced, with each SPD presented first for five of the ten evaluations. Hence this required a test participant to provide 50 evaluations each for the chromatic and achromatic conditions. The five comparisons were carried out randomly within the block of 50 evaluations.

Following Berman et al., the two light sources being compared were identified by the experimenter to the test participant by giving each source a random number (from within the range 1 to 9) and test participants were informed of each source using this number, e.g. 'Here is number 3, here is number 7' repeated three times. The question was then asked 'Which one appeared brighter?'. Responses were recorded by the experimenter with bespoke software to display the stimuli and record the answers. The last presentation remained on while the question was asked and until the next sequence began, approximately 6-7 seconds later. The instructions were clarified as follows: By brightness we 
mean the amount of light in the booth, ignoring any colour differences between lights and surfaces. The two different number labels for the lights in each pair are taken randomly from the range 1 to 9 . When a number comes up again it does not mean the same light as in a previous pair; judge the current lights only; try to avoid being influenced by memory of previous lights.'

\subsection{Procedure II: Fotios and Cheal matching}

The second procedure follows that used by Fotios and $\mathrm{Cheal}^{26}$ who examined spatial brightness at mesopic levels of adaptation, but using sequential matching in a single booth rather than simultaneous matching in side-by-side booths. It was not expected that this difference would significantly affect the results. ${ }^{31}$ For concurrent validation of the matching results a brightness discrimination task was included within the procedure, this being the third procedure of the current study.

Light settings were seen in pairs, presented sequentially. Each light source was presented for 5 seconds, with three presentations of each light source separated by a $100 \mathrm{~ms}$ dark interval. One SPD was presented at the reference luminance $\left(50 \mathrm{~cd} / \mathrm{m}^{2}\right)$ and the luminance of the second SPD was adjusted by the participant until the two appeared, as near as possible, equally bright. As above, brightness was described as the amount of light in the whole scene which could be judged independently from any other visual differences such as colour. Each test participant provided four brightness matches for the three between-SPD pairs and two matches for the null condition, counterbalancing both the initial luminance of the variable stimulus (set by the experimenter to a level clearly higher or lower than the reference, luminances of $75 \mathrm{~cd} / \mathrm{m}^{2}$ and $25 \mathrm{~cd} / \mathrm{m}^{2}$, respectively) and the application of dimming to both sources. These trials were carried out in a random order.

\subsection{Procedure II: Fotios and Cheal discrimination}

For discrimination judgements, two SPDs were presented in alternation and test participants instructed to state which was the brighter, a forced-choice procedure with the equally bright response option not permitted. Each light source was presented for 5 seconds, with three presentations of each light source separated by a $100 \mathrm{~ms}$ dark interval. Both SPDs provided the same luminance, $50 \mathrm{~cd} / \mathrm{m}^{2}$. The SPD sequence (first or second) was random within the eight evaluations in each of the achromatic and chromatic environments.

\subsection{Test procedure}

Tests with each participant were completed in a single 2-hour session. Lighting for the initial 10-15 minutes of a test session was provided by SPD A set to $67 \mathrm{~cd} / \mathrm{m}^{2}$. In this time the participant was given instructions for the test procedure and completed a distraction test of attempting to place the FM-100 colour chips into the correct order. The first lighting condition for the experiment was presented following this adaptation.

For a given SPD pair, six steps were carried out, the order being counterbalanced between test participants:

1) Berman et al. discrimination task with achromatic environment.

2) Berman et al. discrimination task with chromatic environment.

3) Fotios and Cheal discrimination task with achromatic environment.

4) Fotios and Cheal discrimination task with chromatic environment.

5) Fotios and Cheal matching task with achromatic environment.

6) Fotios and Cheal matching task with chromatic environment.

The Berman et al. task was carried out using two SPDs (A and B), the aim being to 
replicate their work. For the Fotios and Cheal task the third SPD was introduced (C) and the three SPDs were presented in all three possible pairs (i.e. $\mathrm{A} / \mathrm{B}, \mathrm{A} / \mathrm{C}$ and $\mathrm{B} / \mathrm{C}$ ).

In the Berman et al. task one null condition was presented (comparison 5), in which the two lighting conditions were identical (SPD A, $47 \mathrm{~cd} / \mathrm{m}^{2}$ ). In the Fotios and Cheal discrimination trials the null condition was SPD $A$ at $50 \mathrm{~cd} / \mathrm{m}^{2}$. SPD A was used also for the matching task null condition, with the reference set to $50 \mathrm{~cd} / \mathrm{m}^{2}$ and the variable source set to starting luminances of 25 or $75 \mathrm{~cd} / \mathrm{m}^{2}$.

Within a test session, the matching and discrimination trials were carried out as separate blocks, the order of these being balanced. Within the discrimination block, the Berman et al. procedure and Fotios and Cheal procedure were carried out in a balanced order. The three procedures were used with both achromatic and coloured interior surfaces, the order being balanced. SPD pairs were presented in an order that was randomised between participants.

28 test participants were used. The results of Fotios and Cheal's ${ }^{26}$ mesopic brightness matching data suggest an effect size of approximately $0.8,{ }^{32}$ suggested by Cohen $^{33}$ to be a large effect, for which a sample of 28 is sufficient to detect the standard level of probability of mistakenly rejecting the null hypothesis $(\alpha=0.05)$ with a power of $0.80 .^{34}$ This sample exceeded those used by Berman et al. $(\mathrm{n}=12)$ and Fotios and Cheal $(\mathrm{n}=21)$ and the demands of the variance stable rank sums method for analysing data from the Fotios and Cheal discrimination procedure and judgements which required 17 test participants to insure the possibility of the three SPD being significantly different at an alpha level of $0.01 .{ }^{35}$ All test participants were confirmed as having colour-normal vision using the Ishihara test. Fourteen were male and 14 were female, and their ages were in the range of 22 to 42 years.

\section{Results}

\subsection{Procedure I: Berman et al.}

The results of trials carried out using the Berman et al. procedure are shown in Table 4. For each comparison there were 280 trials ( 28 participants $\times 10$ repeats): Table 4 shows the total frequencies by which each of a pair of stimuli were considered to be brighter summated for all test participants and also the mean and median frequencies per participant. For a given pair, a count of 140 votes per SPD would indicate equal brightness. This is the result that tended to be found for comparisons 1 and 2 except for a larger difference in comparison 2 achromatic. In three cases there is a tendency for SPD B to be considered the brighter but in comparison 1 chromatic, SPD A was considered to be the brighter.

First consider the control and null condition results (comparisons 4 and 5). When the two stimuli were identical (luminance and SPD) there was an approximately equal frequency of votes for each of them (comparison 5), suggesting negligible interval bias. These data are repeated measures and are not drawn from a normally distributed population. Analysis using the Wilcoxon signedranks test did not suggest differences between the first and second intervals to be significant, for trials with either the chromatic or achromatic environments.

When one stimulus was of higher luminance than the other, the SPDs being equal (comparison 4), then there was a near $100 \%$ frequency for the higher luminance to be considered the brighter. This difference was confirmed to be significant for both the chromatic and achromatic environments using the Wilcoxon test $(p<0.01)$, suggesting that the test was of sufficient sensitivity.

The result of comparison 4 is of interest because the luminance ratio presented is identical to that used in comparisons 1 and 2 where the SPDs of the two settings were 
Table 4 Results of the discrimination trials following the Berman et al. procedure. These data are the average frequencies by which each of a pair of stimuli were considered to be brighter. For each comparison there were 280 trials (28 participants $\times 10$ repeats)

\begin{tabular}{|c|c|c|c|c|c|c|c|c|c|c|}
\hline & \multicolumn{2}{|c|}{ Comparison 1} & \multicolumn{2}{|c|}{ Comparison 2} & \multicolumn{2}{|c|}{ Comparison 3} & \multicolumn{2}{|c|}{ Comparison 4} & \multicolumn{2}{|c|}{ Comparison 5} \\
\hline & $\mathrm{A} 47^{\mathrm{a}}$ & B40 & A67 & B57 & A67 & B40 & B67 & B57 & A47 (1st) & A47 (2nd) \\
\hline \multicolumn{11}{|l|}{ Achromatic } \\
\hline TOTAL & 135 & 145 & 97 & 183 & 264 & 16 & 268 & 12 & 146 & 134 \\
\hline Mean & 4.8 & 5.2 & 3.5 & 6.5 & 9.4 & 0.6 & 9.6 & 0.4 & 5.2 & 4.8 \\
\hline Std. Dev. & 2.96 & 2.99 & 2.55 & 2.55 & 1.83 & 1.83 & 1.35 & 1.35 & 1.89 & 1.89 \\
\hline $\begin{array}{l}\text { Median } \\
\text { Chromatic }\end{array}$ & 5 & 5 & 3.5 & 6.5 & 10 & 0 & 10 & 0 & 5.5 & 4.5 \\
\hline TOTAL & 159 & 121 & 129 & 151 & 257 & 23 & 275 & 5 & 141 & 139 \\
\hline Mean & 5.7 & 4.3 & 4.5 & 5.4 & 9.2 & 0.8 & 9.8 & 0.2 & 5.0 & 5.0 \\
\hline Std. Dev. & 3.29 & 3.29 & 3.16 & 3.14 & 2.02 & 2.02 & 0.48 & 0.48 & 1.43 & 1.43 \\
\hline Median & 6 & 4 & 4 & 6 & 10 & 0 & 10 & 0 & 5 & 5 \\
\hline
\end{tabular}

${ }^{\mathrm{a}} \mathrm{A} 47$ denotes SPD A with a luminance of $47 \mathrm{~cd} / \mathrm{m}^{2}$.

different, with the SPD of higher $\mathrm{S} / \mathrm{P}$ ratio (B) being presented at the lower luminance. Following Berman et al., an effect of SPD was investigated by contrasting comparison 1 with comparison 4 (and similarly 2 with 4 ). The differences were confirmed to be significant for both the chromatic and achromatic environments using the Wilcoxon test $(p<0.01$ for all four cases). This demonstrates that the higher $\mathrm{S} / \mathrm{P}$ ratio of setting $\mathrm{B}$ led to fewer reports that setting A was the brighter.

Comparisons 1 and 2 provided the same ratio (higher/lower) of photopic luminances and the same SPD pairs, but comparison 2 was carried out at a higher absolute luminance than comparison 1. Differences between comparison 1 and comparison 2 are suggested to be significant $(p<0.01)$ using the Wilcoxon test. For both the achromatic and chromatic data, at the higher luminance (comparison 2) there was a higher frequency of reports that the high $\mathrm{S} / \mathrm{P}$ ratio setting (B) was brighter: At the lower luminance, the two settings were of near equal brightness in the achromatic environment and the high $\mathrm{S} / \mathrm{P}$ ratio source was considered to be the dimmer in the chromatic environment. These data suggest that relative luminances required for equal brightness vary with absolute luminance. While this disagrees with past studies of spatial brightness at photopic levels ${ }^{11,36}$ and at mesopic levels, ${ }^{37}$ there is recent evidence from studies at mesopic levels suggesting that spectral sensitivity for spatial brightness might differ for different light levels: ${ }^{38,39}$ further research is needed to evaluate this.

In comparison 3, the SPD of higher $\mathrm{S} / \mathrm{P}$ ratio (B) was again presented at the lower luminance, but the difference was much larger than in comparisons 1 and 2 . The results demonstrate a near $100 \%$ frequency for the SPD of higher luminance to be seen as brighter. What comparison 3 shows is that if higher $\mathrm{S} / \mathrm{P}$ ratio does lead to higher spatial brightness, there is a limit to the effect, as at some point the majority of responses are for the setting of higher luminance regardless of the $\mathrm{S} / \mathrm{P}$ ratio. Figure 3 shows the proportion of votes for a particular source to be brighter plotted against the ratio of photopic luminances, and these six points are for comparisons 1,2 and 3 for the achromatic and chromatic environments. A response proportion of 0.5 indicates the two SPDs were considered equally bright, and in Figure 3 this 


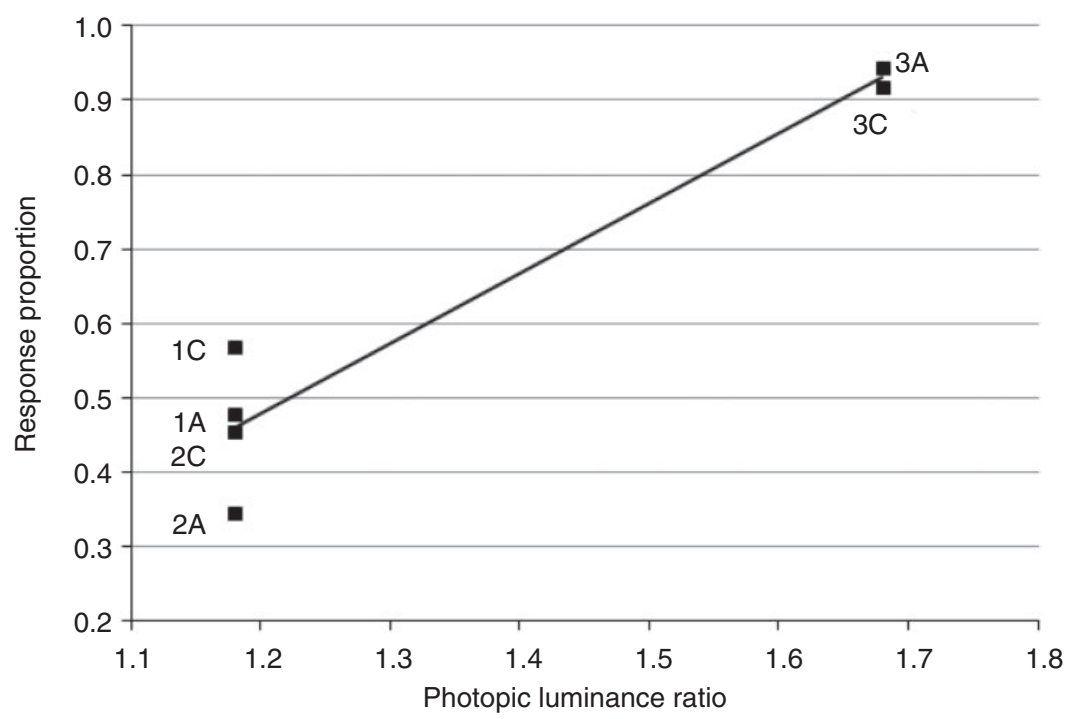

Figure 3 The proportion of votes for SPD A to be brighter than SPD B plotted against the ratio of photopic luminances. Note: ' $1 C^{\prime}$ indicates comparison 1 with the chromatic environment

would be a ratio of photopic luminances of $\mathrm{A} / \mathrm{B}=1.22$. If the two environments are considered separately then this ratio would be 1.26 for the achromatic environment and 1.16 for the chromatic environment.

Brightness Lumens (Equation 1) predicted that these two sources would be equally bright with a luminance ratio of 1.31 , a slightly higher ratio than found in these results. For these data, changing the index in Equation 1 from 0.5 to 0.36 (i.e. Brightness lumens $\left.=\mathrm{P}(\mathrm{S} / \mathrm{P})^{0.36}\right)$ provides the prediction of luminances for equal brightness. We do not claim that 0.36 is the more correct value, and when fitting brightness lumens to the results of past studies found that 0.56 was the optimum value. ${ }^{15}$ Instead, this difference indicates the variability found in brightness responses and that $\mathrm{S} / \mathrm{P}$ ratio alone may be insufficient to predict relative spatial brightness.

Figure 3 shows that the proportions of test participants considering SPD A to be the brighter are similar for the achromatic and chromatic environments in comparison 3 with a larger difference for comparisons 1 and 2 . Results of comparisons 4 and 5 in Table 4 do not suggest a difference. The Wilcoxon test suggested differences between the chromatic and achromatic environments to be near significant for comparison $1(p=0.059)$ and significant for comparison $2(p=0.05)$ but did not suggest differences in comparison 3,4 or 5 to be significant $(p \approx 0.50)$. This pattern may be as expected: when the settings are of identical SPD (4 and 5) or when the difference in luminance is large (3 and 4) then addition of the coloured Mondrian pattern made little difference, but when the judgement was made more difficult by using settings of different SPD and little difference in brightness, then the coloured surface had an effect.

\subsection{Procedure II: Fotios and Cheal matching}

3.2.1. Null condition results

Within the matching procedure there were four null condition trials. Two identical SPDs (A) were matched with the variable SPD starting from either a higher $\left(75 \mathrm{~cd} / \mathrm{m}^{2}\right)$ or lower $\left(25 \mathrm{~cd} / \mathrm{m}^{2}\right)$ luminance than the reference 
Table 5 Results of null condition trials in the matching procedure

\begin{tabular}{lllllll}
\hline & Achromatic & & & \multicolumn{2}{l}{ Chromatic } & \\
\cline { 2 - 3 } & Start high & Start lown & & Start high & Start low \\
\hline Mean luminance ratio & 0.98 & 1.02 & & 1.00 & 1.01 \\
Std. Dev & 0.06 & 0.06 & & 0.08 & 0.10 \\
N & 28 & & & & & 28 \\
Difference from unity (t-test) & n.s. & n.s. & & n.s. & n.s. \\
\hline
\end{tabular}

n.s. $=$ not statistically significant, $p>0.05$.

Table 6 Results of the brightness matching tests: Mean luminance ratios at equal brightness ( $\mathrm{n}=28$, all cases)

\begin{tabular}{|c|c|c|c|c|c|c|c|}
\hline \multirow[t]{2}{*}{ Test condition } & & \multicolumn{3}{|c|}{ Achromatic } & \multicolumn{3}{|c|}{ Chromatic } \\
\hline & & $A / B$ & $\mathrm{~A} / \mathrm{C}$ & $\mathrm{B} / \mathrm{C}$ & $A / B$ & $\mathrm{~A} / \mathrm{C}$ & $\mathrm{B} / \mathrm{C}$ \\
\hline \multirow{2}{*}{ 1st dimmed, start high } & Mean luminance ratio & 1.17 & 1.44 & 1.11 & 1.21 & 1.42 & 1.17 \\
\hline & Std. Dev. & 0.21 & 0.24 & 0.17 & 0.23 & 0.25 & 0.21 \\
\hline \multirow[t]{2}{*}{ 1st dimmed, start low } & Mean luminance ratio & 1.18 & 1.32 & 1.11 & 1.16 & 1.40 & 1.13 \\
\hline & Std. Dev. & 0.17 & 0.21 & 0.17 & 0.19 & 0.19 & 0.18 \\
\hline \multirow[t]{2}{*}{ 2nd dimmed, start high } & Mean luminance ratio & 1.19 & 1.23 & 1.14 & 1.18 & 1.39 & 1.19 \\
\hline & Std. Dev. & 0.14 & 0.15 & 1.20 & 0.17 & 0.22 & 0.25 \\
\hline \multirow[t]{2}{*}{ 2nd dimmed, start low } & Mean luminance ratio & 1.19 & 1.30 & 1.16 & 1.22 & 1.45 & 1.20 \\
\hline & Std. Dev. & 0.12 & 0.20 & 0.16 & 0.17 & 0.30 & 0.29 \\
\hline \multirow[t]{3}{*}{ Overall } & Mean luminance ratio & 1.18 & 1.32 & 1.13 & 1.19 & 1.41 & 1.17 \\
\hline & Std. Dev. & 0.10 & 0.14 & 0.10 & 0.13 & 0.17 & 0.14 \\
\hline & $\begin{array}{l}\text { Difference from } \\
\text { unity (t-test) }\end{array}$ & $p<0.01$ & $p<0.01$ & $p<0.01$ & $p<0.01$ & $p<0.01$ & $p<0.01$ \\
\hline
\end{tabular}

$\left(50 \mathrm{~cd} / \mathrm{m}^{2}\right)$, and this with the achromatic and chromatic environments. As shown in Table 5, the mean luminance ratios (fixed/ variable) ranged from 0.98 to 1.02 in these four cases.

These data were considered to be normally distributed following analysis using measures of central tendency, dispersion, graphical presentation and statistical analysis (ShapiroWilks, Kolmogorov-Smirnov). Application of the t-test does not suggest these mean luminance ratios to depart significantly from unity in any of the four conditions and thus that interval bias was negligible.

The results do demonstrate an anchor effect: When the variable source started from a low luminance the equal brightness luminance is lower than that found when starting from a high luminance. While a similar anchoring effect has been found in some past studies, ${ }^{36,37}$ a significant effect in the opposite direction has also been found. ${ }^{40}$ The t-test suggests the anchor effect is near significant $(p=0.064)$ for the achromatic environment but for the chromatic environment did not suggest the difference to be significant $(p=0.63)$. The initial luminances were balanced in trials to offset the effect of anchoring.

\subsubsection{Mixed-SPD results}

In trials, each of the three SPD pairs $(\mathrm{A} / \mathrm{B}$, $\mathrm{A} / \mathrm{C}$ and $\mathrm{B} / \mathrm{C}$ ) was matched four times by each test participant, in order to balance which of the pair was the variable source and whether this started from a higher or lower luminance than that of the reference. This was repeated for the achromatic and chromatic environments. The results of these trials are shown in Table 6, these data being the mean luminance ratio at equal brightness. 
Table 7 Results of brightness discrimination tests: judgements of brighter SPD when presented at equal luminance

\begin{tabular}{|c|c|c|c|c|c|c|c|c|}
\hline & \multicolumn{8}{|c|}{ Frequency for first SPD in each pair to be judged as brighter } \\
\hline & \multicolumn{4}{|c|}{ Achromatic } & \multicolumn{4}{|c|}{ Chromatic } \\
\hline & $A / B$ & $\mathrm{~A} / \mathrm{C}$ & $\mathrm{B} / \mathrm{C}$ & Null & $A / B$ & $\mathrm{~A} / \mathrm{C}$ & $\mathrm{B} / \mathrm{C}$ & Null \\
\hline Forward order $(n=28)$ & 4 & 1 & 6 & 13 & 6 & 0 & 2 & 14 \\
\hline Reverse order $(n=28)$ & 2 & 3 & 6 & 14 & 7 & 1 & 3 & 17 \\
\hline Overall $(n=56)$ Frequency & 6 & 4 & 12 & 27 & 13 & 1 & 5 & 31 \\
\hline Percentage & $10.7 \%$ & $7.3 \%$ & $21.4 \%$ & $49.1 \%$ & $23.2 \%$ & $1.8 \%$ & $8.9 \%$ & $55.4 \%$ \\
\hline Brighter lamp & B & C & C & $=$ & B & C & & $=$ \\
\hline Difference & $p<0.01$ & $p<0.001$ & $p<0.05$ & n.s. & $p<0.05$ & $p<0.001$ & $p<0.001$ & n.s. \\
\hline
\end{tabular}

Note: (1) There was one missing value each in the $A / C$ and null pairs for the achromatic environment.

(2) Forward order means SPD order (1st interval/2nd interval) was $A / B, A / C$ and $B / C$; reverse order means this was $B / A$, $\mathrm{C} / \mathrm{A}$ and $\mathrm{C} / \mathrm{B}$.

Analyses of these distributions revealed 10 outlier values from within the 672 data points, these being distributed across the range of test conditions. Analysis of the distributions with outlying values omitted suggested they were drawn from normally distributed populations. Two-way repeated measures analysis of variance (ANOVA) was applied to examine the effect of SPD order (e.g. whether A of the pair A/B was the first or second to be presented in the sequence) and the effect of initial luminance (i.e. luminance of the variable SPD set to a high or low level prior to the trial) with the outlier values omitted and treated as missing values. ANOVA does not suggest that starting luminance (high or low) led to significant differences in luminance ratio at equal brightness. In only two of the six cases, ANOVA suggests SPD order (1st or 2nd in the sequence) to be significant (A/B chromatic and $\mathrm{A} / \mathrm{C}$ achromatic). In any case, starting luminance and SPD presentation order were counterbalanced within trials to offset the effects of any such bias.

For each test participant, the mean of these four trials was therefore used as the best estimate of their luminance ratio at equal brightness for each combination of SPD pair and interior colour. These data are shown in the 'overall' row in Table 6. Analysis of these merged distributions suggested they were drawn from a normally distributed population, the one outlier found in this set being retained. According to the one-sample t-test, these luminance ratios depart significantly from unity in all six cases $(p<0.01)$, thus demonstrating that SPD has a significant effect on spatial brightness.

The trials carried out with the coloured surfaces inside the booth led to luminance ratios which depart further from unity and with a higher variance than trials with the achromatic surfaces. The effect of adding the coloured surface was examined using the paired samples t-test. For SPD pair A/B the ttest did not suggest a significant difference $(p=0.64)$. The differences were significant for SPD pairs $\mathrm{B} / \mathrm{C}(p<0.05)$ and $\mathrm{A} / \mathrm{C}(p<0.01)$, with the coloured environment leading to luminance ratios that depart further from unity than with the achromatic surface.

\subsection{Procedure III: Fotios and Cheal discrimination}

Results of the brightness discrimination trials are shown in Table 7. These show the frequency of responses in which one lamp in the pair was considered to be brighter.

In null condition trials, identical SPDs were compared at equal luminance, and test participants responded whether the first or 
Table 8 Comparison of the results gained from different test procedures

\begin{tabular}{|c|c|c|c|c|c|c|c|}
\hline \multirow[t]{2}{*}{ Procedure } & \multirow[t]{2}{*}{ Finding } & \multicolumn{3}{|c|}{ Achromatic } & \multicolumn{3}{|c|}{ Chromatic } \\
\hline & & $A / B$ & $\mathrm{~A} / \mathrm{C}$ & $\mathrm{B} / \mathrm{C}$ & $A / B$ & $\mathrm{~A} / \mathrm{C}$ & $\mathrm{B} / \mathrm{C}$ \\
\hline \multirow[t]{2}{*}{ Berman et al. discrimination } & Brighter SPD & B & - & - & B & - & - \\
\hline & $\begin{array}{l}\text { Luminance ratio for equal } \\
\text { brightness }\end{array}$ & 1.26 & - & - & 1.16 & - & - \\
\hline \multirow[t]{3}{*}{ Fotios and Cheal, matching } & Brighter SPD & B & C & C & B & C & C \\
\hline & $\begin{array}{l}\text { Luminance ratio for equal } \\
\text { brightness }\end{array}$ & 1.18 & 1.32 & 1.13 & 1.19 & 1.41 & 1.17 \\
\hline & Std. dev. & 0.10 & 0.14 & 0.10 & 0.13 & 0.17 & 0.14 \\
\hline Fotios and Cheal, discrimination & Brighter SPD & B & C & C & B & C & C \\
\hline
\end{tabular}

second interval was the brighter. The results indicate almost equal frequencies for the first and second intervals (the first interval was reported to be brighter in $49.1 \%$ and $55.4 \%$ of trials for the achromatic and chromatic tests, respectively) and thus that interval bias was negligible. The binomial test did not suggest interval bias to be significant in either case.

It can be seen in Table 7 that the frequency of votes for SPD A in the pair A/B is similar for both presentation orders (i.e. $\mathrm{A} / \mathrm{B}$ and $\mathrm{B} /$ $\mathrm{A})$, and this is also the case for pairs $\mathrm{A} / \mathrm{C}$ and $\mathrm{B} / \mathrm{C}$. Of the 335 discrimination trials (i.e. 336 trials with one missing case) test participants gave different responses in their two trials per SPD pair (i.e. $\mathrm{A} / \mathrm{B}$ and $\mathrm{B} / \mathrm{A}$ ) on only 22 occasions. This suggests that presentation order had negligible effect and in any case this was balanced. Differences between SPDs were examined using Dunn-Rankin Variance Stable Rank Sums. ${ }^{35}$ This analysis suggests that SPD B is brighter than SPD A $(p<0.01$, achromatic; $p<0.05$ chromatic); SPD $\mathrm{C}$ is also brighter than SPD A $(p<0.001$, achromatic and chromatic); SPD $\mathrm{C}$ is brighter than SPD B $(p<0.001$ chromatic, $p<0.05$ achromatic).

\section{Discussion}

This experiment was carried out to compare two potential metrics for the effect of SPD on spatial brightness and to compare different experimental procedures. The results are summarised in Table 8 . It can be seen that the three procedures concur as to which of a pair of SPDs would be considered the brighter at equal luminance. For the A/B pair, luminance ratios for equal brightness were determined using either the matching procedure or interpolated from the Berman et al. discrimination procedure. According to the one sample t-test these are significantly different $(p<0.01)$ for the achromatic environment but are not suggested to be different for the chromatic environment. Further evidence is needed to compare these methods and to determine which provides the more accurate response. What we can confirm is that the matching procedure can be completed in less time, which is why in the current study we did not use the Berman et al. procedure for the remaining two lamp pairs.

This study aimed to repeat, as near as possible, the experiment reported by Berman et $a l .{ }^{17}$ One reason for this replication was that Berman et al. did not include a null condition trial sufficient to evaluate interval bias associated with sequential evaluations the potential tendency for test participants to consistently report one interval (e.g. the second) as being the brighter regardless of the stimuli observed. This is particularly expected in procedures where two stimuli are observed only once each, with the 
judgement made during observation of the second: there is a tendency for memory to recall the first interval as being darker than it was, ${ }^{41,42}$ thus enhancing the frequency by which the second interval is reported to be brighter. Berman et al. used a sequential evaluation where each stimulus was presented three times, and a review ${ }^{29}$ of these data asked whether this repeated sequential presentation was sufficient to counter interval bias. In the current study, comparison 5 was included to examine this, being a null condition where both settings were of equal luminance and SPD. Analysis of these data did not find a difference between the two intervals which suggests that three sequential observations of each SPD in alternation are sufficient to offset the interval bias associated with successive evaluation.

SPD pair A/B were of equal chromaticity but different $\mathrm{S} / \mathrm{P}$ ratio. Interpolation of the results suggest significant difference in spatial brightness at equal luminance, thus confirming that in this case the higher $\mathrm{S} / \mathrm{P}$ ratio led to higher spatial brightness. SPD pair B/C were of similar $\mathrm{S} / \mathrm{P}$ ratio but different gamut area, and the results demonstrate that the source of higher gamut area was significantly brighter at equal luminance.

For sources of equal chromaticity, the $\mathrm{S} / \mathrm{P}$ ratio matters, providing support for the conclusion drawn by Berman et al. ${ }^{17}$ If, instead, the $\mathrm{S} / \mathrm{P}$ ratio is held constant, then gamut area matters - or rather, the spatial brightness response characterised by gamut area in this study matters. SPD pair A/C presented differences in $\mathrm{S} / \mathrm{P}$ ratio and gamut area, and here the source of higher $\mathrm{S} / \mathrm{P}$ ratio and gamut area was found to be significantly brighter. Pair $\mathrm{A} / \mathrm{C}$ indicates that both $\mathrm{S} / \mathrm{P}$ ratio and gamut area matter when neither is held constant, and thus that better prediction of relative spatial brightness would be found by considering both metrics simultaneously. What is interesting here is that transitivity holds: Within the achromatic and chromatic results individually, the product of $\mathrm{A} / \mathrm{B}$ and $\mathrm{B} / \mathrm{C}$ provides good agreement for the finding of $\mathrm{A} / \mathrm{C}$. If one effect (i.e. $\mathrm{S} / \mathrm{P}$ ratio or gamut area) were dominant, then assumption of transitivity from $\mathrm{A} / \mathrm{B}$ and $\mathrm{B} / \mathrm{C}$ would tend to over-estimate the result for $\mathrm{A} / \mathrm{C}$.

These results suggest that lighting of higher $\mathrm{S} / \mathrm{P}$ ratio appears brighter, which disagrees with past results. ${ }^{43}$ Similarly, past research at mesopic levels found that gamut area (and a model based on the short wavelength sensitive cone, the SWS/P ratio) exhibited weaker correlation with test results than did the other metrics examined including $\mathrm{S} / \mathrm{P}$ ratio. ${ }^{26}$ One possible explanation is that the $\mathrm{S} / \mathrm{P}$ ratio and gamut area reduce the complex SPD of a light source to a single number, and this act of data reduction loses relevant information. Royer and Houser ${ }^{27}$ examined a large range of such metrics and concluded that none of them provided a satisfactory explanation for spatial brightness.

Two studies have suggested the need for a dual-metric approach to characterising colourrendering qualities, comprising a gamut-based metric and a reference-based metric, e.g. gamut area and $R_{a} \cdot{ }^{44,45}$ It may be found that such an approach is of also benefit for predicting spatial brightness, improving the reliability of prediction. Note that a literature review of spatial brightness concluded that consideration of CCT and $\mathrm{R}_{\mathrm{a}}$ together provided a more reliable prediction of SPD effects than did either CCT or CRI alone. ${ }^{46}$

Clearly, these results are not confirmation that $\mathrm{S} / \mathrm{P}$ ratio and gamut area are the optimum metrics. It may be found that the s-cone or ipRGC response is more appropriate than the scotopic component of the $\mathrm{S} / \mathrm{P}$ ratio, and current activity regarding colour rendering may establish a better metric than gamut area.

The results suggest some differences between brightness evaluations made in the chromatic and achromatic environments. According to the matching test, the difference is significant for SPD pairs $A / C$ and $B / C$, 
these having different chromaticities, but not for A/B which had similar chromaticity. This disagrees with the findings of past experiments that the colour of surfaces in an environment did not affect evaluations of spatial brightness. ${ }^{11,12,28,47}$ One reason may be that in the current study the test participant was placed at a relatively short distance from the booth surfaces and this may have led to evaluations of the surface rather than of the illuminated volume. We aim to address this in further work by repeating the experiment using a larger test environment.

\section{Conclusion}

This paper describes an experiment carried out to investigate the influence of lamp SPD on spatial brightness using three different procedures. The discrimination procedure used by Berman et al. ${ }^{17}$ was validated through inclusion of an additional null condition to evaluate interval bias and through parallel use of alternate procedures, the matching and discrimination procedures used by Fotios and Cheal. ${ }^{26}$ These different procedures provided converging evidence as to which of a pair of SPDs is the brighter, and provided similar estimates as to the magnitude of the effect.

This paper provides further support for the conclusion reported by Berman et al. that for two lights of equal chromaticity and equal luminance, the one of higher $\mathrm{S} / \mathrm{P}$ ratio will appear brighter. Berman et al. used only two SPDs to test this proposal. In the current work a third SPD was added in order to evaluate the impact of a chromatic contribution to spatial brightness for two lights of equal $\mathrm{S} / \mathrm{P}$ ratio. The results suggest that both the $\mathrm{S} / \mathrm{P}$ ratio and the chromatic contribution are important and that considering both metrics simultaneously enables a better prediction of spatial brightness under different SPDs. In other words, the results suggest that consideration of either $\mathrm{S} / \mathrm{P}$ ratio or gamut area alone would be insufficient. The current study investigated the $\mathrm{S} / \mathrm{P}$ ratio and gamut area as past work identified these as potential metrics for spatial brightness. This does mean that they are the correct metrics to use in the first place, but the current study provides some further validation, and as established metrics of SPD their familiarity may be advantageous when consideration is given to inclusion in design guidance.

\section{Funding}

Naoya Hara's contribution to this work was funded by the Kansai University's Overseas Research Program for the year of 2012.

\section{References}

1 Boyce P. Tactics or strategy? Lighting Research and Technology 2013; 45: 519.

2 Boyce P. Lighting under pressure. Lighting Research and Technology 2010; 42: 5.

3 Tregenza P, Loe D. The Design of Lighting. London: Spon Press, 1998.

4 Boyce PR. Human Factors in Lighting, 2nd Edition. London: Taylor and Francis, 2003.

5 Rea MS. Practical implications of a new visual performance model. Lighting Research and Technology 1986; 18: 113-118.

6 Society for Light and Lighting. Code for Lighting. Oxford: Butterworth-Heinemann, 2002.

7 Ramasoot T, Fotios SA. Lighting and display screens: Models for predicting luminance limits and disturbance. Lighting Research and Technology 2012; 44: 197-223.

8 Fotios S, Atli D. Comparing judgements of visual clarity and spatial brightness through an analysis of studies using the category rating procedure. Leukos 2012; 8: 261-281.

9 Fotios S, Atli D, Cheal C, Houser K, Logadottir A. Lamp spectrum and spatial brightness at photopic levels: A basis for developing a metric. Lighting Research and Technology. First published: 20 September, 2013. DOI $10.1177 / 1477153513503170$. 
10 Akashi Y, Boyce PR. A field study of illuminance reduction. Energy and Buildings 2006; 38: 588-599.

11 Boyce PR. Investigations of the subjective balance between illuminance and lamp colour properties. Lighting Research and Technology 1977; 9: 11-24.

12 Boyce PR, Cuttle C. Effect of correlated colour temperature on the perception of interiors and colour discrimination. Lighting Research and Technology 1990; 22: 19-36.

$13 \mathrm{Hu}$ X, Houser KW, Tiller DK. Higher colour temperature lamps may not appear brighter. Leukos 2006; 3: 69-81.

14 Ware C, Cowan WB. Specification of Heterochromatic Brightness Matches: A Conversion Factor for Calculating Luminances of Stimuli that are Equal in Brightness. National Research Council Canada. NRC Publication Number 26055, Ottawa: NRC, 1983.

15 Fotios S, Atli D, Cheal C. Comparing metrics for relative spatial brightness under lamps of different spectral power: Proceedings of Lux Pacifica, Bangkok, March 6-8: 2013: 209-212.

16 Commission Internationale de l'Éclairage. CIE Report 206:2014. The Effect of Spectral Power Distribution on Lighting for Urban and Pedestrian Areas. Vienna: CIE, 2014.

17 Berman SM, Jewett DL, Fein G, Saika G, Ashford F. Photopic luminance does not always predict perceived room brightness. Lighting Research and Technology 1990; 22: 37-41.

18 Berman SM. Correspondence. A new retinal photoreceptor should affect lighting practise. Lighting Research and Technology 2008; 40: 373-376.

19 Brown TM, Tsujimura S, Allen AE, Wynne J, Bedford R, Vickery G, Vugler A, Lucas RJ. Melanopsin-based brightness discrimination in mice and humans. Current Biology 2012; 22: $1-8$.

20 Illuminating Engineering Society of North America. IES TM-24-13. An Optional Method for Adjusting the Recommended Illuminance for Visually Demanding Tasks Within IES Illuminance Categories P through Y Based on Light Source Spectrum. New York: IESNA, 2013.
21 Hunt RWG. Measuring Colour, 2nd Edition. London: Ellis Horwood, 1995.

22 Lennie P, Pokorny J, Smith VC. Luminance. Journal of the Optical Society of America (A) 1993; 10: 1283-1293.

23 Wagner G, Boynton RM. Comparison of four methods of heterochromatic photometry. Journal of the Optical Society of America 1972; 62: 1508-1515.

24 Yaguchi H, Ikeda M. Contribution of opponent-colour channels to brightness. In: Mollon JD, Sharpe LT. (eds) Colour Vision: Physiology and Psychophysics. London: Academic Press, 1983.

25 Fotios S, Gado T. A comparison of visual objectives used in side-by-side matching tests. Lighting Research and Technology 2005; 37: 117-131.

26 Fotios SA, Cheal C. Predicting lamp spectrum effects at mesopic levels. Part 1: Spatial brightness. Lighting Research and Technology 2011; 43: 143-157.

27 Royer MP, Houser KW. Spatial brightness perception of trichromatic stimuli. Leukos 2012; 9: 89-108.

28 Fotios SA, Cheal C. Brightness matching with visual fields of different types.

Lighting Research and Technology 2011; 43: 73-85.

29 Fotios S, Houser K. Using forced choice discrimination to measure the perceptual response to light of different characteristics. Leukos 2013; 9: 245-259.

30 Berman S. Implications of Rod Sensitivity to Interior Lighting Practice, CIE Publication x009-1995. Vienna: CIE, 171-176.

31 Fotios SA, Cheal C. A comparison of simultaneous and sequential brightness judgements. Lighting Research and Technology 2010; 42: 183-197.

32 Faul F, Erdfelder E, Lang AG, Buchner A. G*Power 3: A flexible statistical power analysis program for the social, behavioral, and biomedical sciences. Behavior Research Methods 2007; 39: 175-191.

33 Cohen J. Statistical power analysis. Current Directions in Psychological Science 1992; 1: 98-101.

34 Field A. Discovering Statistics Using SPSS. Sage Publications: London, 2005. 
35 Dunn-Rankin P, Knezek GA, Wallace S, Zhang S. Scaling Methods, 2nd Edition, Mahway, NJ: Lawrence Erlbaum Associates, 2004.

36 Fotios SA, Levermore GJ. The perception of electric light sources of different colour properties. Lighting Research and Technology 1997; 29: 161-171.

37 Fotios SA, Cheal C. Lighting in subsidiary streets: Investigation of SPD effects. Part 2 Brightness. Lighting Research and Technology 2007; 39: 233-252.

38 Rea MS, Radetsky LC, Bullough JD. Toward a model of outdoor lighting scene brightness. Lighting Research and Technology 2011; 43: 7-24.

39 Bullough JD, Radetsky LC, Besenecker UC, Rea MS. Influence of spectral power distribution on scene brightness at different light levels. Leukos 2014; 10: 3-9.

40 Houser KW, Tiller DK, Hu X. Prototype demonstration of vision-tuned fluorescent lamps. Final Report for California Energy Commission. Sacramento, CA: CAC, 2003.

41 Uchikawa K, Ikeda M. Accuracy of memory for brightness of colored lights measured with successive comparison method. Journal of the Optical Society of America A 1986; 3: 34-39.
42 LaBoeuf RA, Shafir E. The long and short of it: Physical anchoring effects. Journal of Behavioural Decision Making 2006; 19: 393-406.

43 Houser KW, Fotios SA, Royer MP. A test of the $\mathrm{S} / \mathrm{P}$ ratio as a correlate for brightness perception using rapid-sequential and side-byside experimental protocols. Leukos 2009; 6: 119-137.

44 Guo X, Houser KW. A review of colour rendering indices and their application to commercial light sources. Lighting Research and Technology 2004; 36: 183-200.

45 Rea MS, Freyssinier-Nova JP. Color rendering: A tale of two metrics. Color Research and Application 2008; 33: 192-202.

46 Fotios SA. Lamp colour properties and apparent brightness: A review. Lighting Research and Technology 2001; 33: 163-181.

47 Han S, Boyce PR. Illuminance, CCT, décor and the Kruithof curve: Proceedings of the 25th Session of the CIE, San Diego, 25 June to 2 July 2003. Vienna: CIE: D3 282-285 (see also Han S. Effect of illuminance, CCT and décor on the perception of lighting. MS thesis, Rensselaer Polytechnic Institute, Troy, NY. 2002.). 